\title{
Bioanalysis
}

\section{Bioanalytical outsourcing: transitioning from Pharma to CRO}

\author{
"As the biopharmaceutical industry continues to evolve, the need \\ to maximize the value of internal operations and maximize the \\ use of research and development dollars is paramount to fostering \\ innovation."
}

First draft submitted: 19 March 2017; Accepted for publication: 11 May 2017; Published online: 1 August 2017

\begin{abstract}
Having spent three quarters of my career at large Pharma performing bioanalysis (BA) inhouse to now working at a large CRO doing BA for small and large Pharma sponsors, the transition has been rapid and dramatic. I can now confidently state that I have seen more drug candidates in the past 20 months than I saw in 20 years while working within Pharma. We used to pride ourselves on delivering at least a double handful of new chemical entities each year into early drug development; however, the unfortunate aspect of this is that we would then watch most of these candidates go pear shaped in the very next phase of drug development. If I was a cynical individual, I might suggest that the quality of drug candidates coming through our $\mathrm{CRO}$ today is not that much better than those of years past. Perhaps the safety and efficacy standards have increased as our ability to detect liabilities has improved or perhaps litigants have proliferated and the risk-benefit equation has become unbalanced.
\end{abstract}

Rather than debate the success or failure of one or the other Pharma's approach to drug development, let us move on to something imminently more interesting, like regulated BA. Know that large Pharma has always outsourced BA, particularly for late-stage clinical programs, wherein sample numbers would overwhelm the internal laboratory which was fundamentally designed for responsiveness but not necessarily routine (less exciting) work. In the early days, we believed that the best scientists were within our own walls and the CROs were sufficiently adequate while being an easy venue to attract competent laboratory staff to join our hallowed staff and perform that 'routine' work. You kept the challenging work inside to stimulate the minds of your world-class scientists and once the problems were solved, you transferred the methods to an external partner or the production team within your own establishment. At best, outsourcing to CROs was a means of overcoming the overflow of in-house capabilities while you built up internal capacity to meet future demand. For the CRO, the expectation was to follow the transferred method without deviation.

Then, procurement groups sniffed out opportunities to commoditize BA and study by study, phase by phase, the work was increasingly outsourced to various vendors. Entire core facilities were dismantled and functions that were once kept in-house are today being performed by any number of an expanding array of contract partners. An exodus of scientific staff from Pharma bioanalytical laboratories to CROs ensued and turned into a flood in the late nineties. Today, it would be a reasonable estimate to state that more than $85 \%$ of the regulated $\mathrm{BA}$ is now performed by a CRO partner. Companies rely on CROs for the expertise that they now lack internally. Consulting is a core business for CROs and a full service one can easily assist in the management of a pipeline candidate through early development and on to proof-of-concept clinical trials. The thought of outsourcing the management of an entire company's portfolio can easily be contemplated. The Biotech community is leading this paradigm shift, as many
Roger Hayes

MPI Research, 54943 North Main Street, Mattawan, MI 49071, USA Tel.: +1 2696683336 Ext.1411 roger.hayes@mpiresearch.com 
of them, it would be fair to say, do not have the talent, expertise or experience that can now be found within a large CRO.

There will always be internal BA performed by large Pharma because the folks sleeping under their own coffins have conditioned their management into believing that responsiveness is sufficient justification to maintain a core bioanalytical function internally. What is often whispered is that a CRO could never respond rapidly to a scientific question because there are all those contracts to be signed, test article to be shipped, methods to be transferred, and not to forget, resource scheduling that gets in the way of that quick answer that will decide the fate of a multibillion dollar asset. Nonetheless, a Pharma BA laboratory may have one new headcount requisition every other year being justified whereas a BA CRO like MPI Research (MI, USA) has at least 20 headcount requisitions to fill at any given time. Meanwhile, our bioanalytical groups continue to double in size at least every 18 months with the large molecule regulated BA group doubling at an even faster frequency. Interestingly, what often remains of a once vibrant large Pharma BA department is a small group of very experienced CRO laboratory monitors whose responsibilities have increased exponentially as procurement has cultivated the symbiotic relationship between the Pharma and the CRO. Their value proposition is easily recognized and understood.

\section{"Although an entrepreneur may not be keen to understand that time flows differently for most of us, upfront, consistent and thorough communication still remains of utmost importance."}

But there are much more than larger Biotech and Pharma developing drug candidates to cure the next unmet medical need. Indeed, MPI Research has more than a hundred new Biotech companies requesting our services each and every year. Almost to a company, none of them have the desire, let alone resources, to build an internal bioanalytical function favoring instead to rely on a CRO partner to fulfill this aspect of their drug development pathway. Now, it gets interesting. A chemist, a druggist, a lawyer and a banker may have successfully founded Coca$\mathrm{Cola}^{\circledR}$; but this quartet of entrepreneurs continues to uncover remarkable drug candidates today and each member of the quartet has a very different concept of space-time than most other folks, certainly those that have to respectfully inform their Sponsor that bioanalytical data will not be available before the animals are placed on study let alone, being dosed.

\section{Considering a career within a bioanalytical CRO?}

To consider the transition from large Pharma to a CRO may have some individuals question their sanity; why would anyone want to leave the comfort of a wellequipped large Pharma laboratory with no more than a double handful of drug candidates to develop each year for the oftentimes frenetic pace of work at a CRO? That is, not unless you were invited to leave the nest as a consequence of the next cost synergy initiative. So, some scientists may feel constrained within the CRO environment; being limited by stringent regulatory guidelines, the endless drive for efficiency (some might call that profitability), timeline pressures, and on occasion even having restricted assay platform choices because of global availability of a particular platform. Sure, while freedom is diminished in comparison to an academic scenario or an innovator discovery department, the insight gained into the diversity, novelty and direction of the marketplace allows for a breadth of experience that is unparalleled in other environments. Working with an antiviral, an adalimumab biosimilar, an ADC and a fusion protein all within weeks of each other allows a depth of scientific exposure that may take years to gain elsewhere. This benefits not only the CRO scientist but quite obviously the Pharma partner who has access to a team with vast experience for troubleshooting issues that inevitably arise during method installation and routine use. A Pharma partner can go along way with engaging the CRO staff by sharing information about the therapeutic target of the drug candidate and then as the program advances, sharing the clinical outcomes of the studies that were supported. The more a CRO bench chemist is engaged in a project the more fulfilling their work becomes and as a consequence, the quality of their work improves and their tenure at the bench extends.

Partnership versus transactional agreements Let us briefly deliberate on the principles of partnership. Although an entrepreneur may not be keen to understand that time flows differently for most of us, upfront, consistent and thorough communication still remains of utmost importance. For those novice to progressing a drug candidate through early preclinical testing, the earlier that a concrete relationship with the CRO is built, the more time, money and heartache can be spared along the way. Opening the lines of communication early can help mitigate some of the more frustrating delays and inefficiencies that those paving the road have endured. Taking a modicum of extra time to develop, characterize and validate a solid method upfront that will be transferable across species, from animal testing to the clinic, will ultimately save time 
and resources while providing data continuity across multiple testing phases. But there are still those Sponsors that are comfortable or at least willing to 'start a study at risk' to potentially shave a few days off of an Investigational New Drug submission date. The usual scenario has the in-life phase initiated without bioanalytical methods being validated or in rare circumstances, without even being developed. The obvious risk is that the exposure assessment may not be reliable particularly if the collection procedures compromise the analyte necessitating a complete redose of the study. This risk must be communicated to and agreed upon by the anxious sponsor.

\section{Specific outsourcing strategies}

Outsourced BA today seems to come from one of three types of sponsors each with distinct requirements. Virtual companies with no laboratory space or hands-on expertise in bioanalytical seem to be the most common, certainly in the early development space. The relationship is almost purely transactional and the $\mathrm{CRO}$ accepts the majority of the responsibility and accountability for providing an acceptable method. It is a minimum expectation that the $\mathrm{CRO}$ provides clear, concise updates on the progress and may even assume the role of mentor for this type of Sponsor.

The second example is more common for large Pharma sponsors, wherein outsourcing is a strategy to meet company timelines. In these cases, the Sponsor has developed a vast amount of knowledge of the assay and wants the CRO to be a seamless extension of its own laboratory. This has its own challenges because the CRO maintains and trains on its own standard operating procedures (SOPs) which could vary in subtle or even different interpretations from those of the Sponsor. Communication once again is paramount but the content of that communication is quite different from a virtual company because it is now focused on understanding exactly how a process will be implemented, as a typical Pharma Sponsor expects highly detailed, ongoing communication with the CRO.

The third example continues an early model of outsourcing wherein the majority of BA has occurred within the Pharma BA laboratory and the sponsor is now anticipating an onslaught of clinical samples for which there are insufficient resources to support. The sponsor's procurement department is expected to select a fully capable CRO who can sign a contract, receive a transferred method seamlessly and send back reportable data with limited interaction.

What is common to all three models is the sponsor expectation that the contracted activities are performed by well-trained bioanalytical scientists. The CRO must provide each bench chemist the training, mentoring and oversight to ensure that the work they perform is executed to appropriate quality standards. Because efficiency is also a key requirement of CRO operations, harmonizing techniques and streamlining bioanalytical processes across the laboratory will be ingrained in the operating principles of the laboratory if the business is to have longevity and be successful.

"Each reinvention has the potential to invalidate or minimally put into question the previous work when it comes time to reconcile the bioanalytical methods at the time of submission."

\section{One-stop-shop or a niche CRO}

There is a continuum to bioanalytical testing initiating with proof-of-concept pharmacology studies, followed by preclinical toxicology safety studies and then on to first-in-human studies after having submitted the Investigational New Drug application. Once a successful Phase I trial is complete, it is on to Phase IIa, Phase IIb and Phase III trials. A large Pharma will likely have a development team handling the different phases and as a consequence, the bioanalytical testing can be managed by different procurement groups. On the other hand, a virtual company will likely not have individual expertise in niche areas of development, such as bioanalytical, and so the company frequently relies on consultants to support the different segments of development. As such, the bioanalytical testing may be performed by many laboratories and individuals as the drug moves through the development process. For example, many toxicology and preclinical CROs are designed to be niche providers, but very few have the knowledge or expertise to support a one-stop-shop for bioanalytical testing through all phases of human trials. Similarly, a standalone clinical bioanalytical CRO is constructed to process large numbers of human samples and for efficiency reasons may refrain from supporting a handful of rabbit plasma samples. At each phase of the process, the assay is transferred and reinvented by each group, putting its own mark on it before validation. Each reinvention has the potential to invalidate or minimally put into question the previous work when it comes time to reconcile the bioanalytical methods at the time of submission.

\section{Availability of critical reagents}

Now that many of the progenitor biologics are coming off patent, the development of biosimilars and biobetters has received considerable investor attention further salivating the appetites of the entrepreneurs. However, the BA CRO confounds this utopia by the rather inconvenient requirement of needing to procure critical assay-enabling reagents. Often, a Sponsor will 
declare the immediate availability of highly purified, fully characterized and preformulated test article but may not have spared one thought about the process of generating the needed ELISA assay components prior to engaging an outsourcing partner. And as often is the case, there is a request to assess the immunogenicity of the biologic and here the concept of a positive control to establish assay sensitivity is considered something that the BA CRO made up to cover our inability to move heaven and Earth to meet Sponsor timelines for the original PK assay. For the early drug development process to advance as smoothly as possible, all parties must understand the critical nature of providing not only acceptable test article in an appropriate formulation but also all of the critical reagents that are required to assess the safety of the drug candidate.

\section{Final thoughts}

The partnering model is all about creating efficiency; allowing a Biopharma company to continue to innovate and discover new therapeutics while operating more efficiently than its competitors. Likewise, the outsourcing partner needs to be an extension of the Sponsor's internal team, and as such, be innovators in their own right. The application of knowledge gained from dealing with a diverse array of Sponsors and their programs affords unrivaled opportunities to the CRO for creative thinking on a new program.

As the biopharmaceutical industry continues to evolve, the need to maximize the value of internal operations and maximize the use of research and development dollars is paramount to fostering innovation. To stay successful, companies are being forced to reevaluate what services/programs they need to maintain in house, and for those that miss the cut, the solution will continue to be enlisting the help of outsourcing partners including academic research organizations, entrepreneurial Biotechs and, of course, CROs.

\section{Financial \& competing interests disclosure}

The author has no relevant affiliations or financial involvement with any organization or entity with a financial interest in or financial conflict with the subject matter or materials discussed in the manuscript. This includes employment, consultancies, honoraria, stock ownership or options, expert testimony, grants or patents received or pending, or royalties.

No writing assistance was utilized in the production of this manuscript.

\section{Open access}

This work is licensed under the Attribution-NonCommercialNoDerivatives 4.0 Unported License. To view a copy of this license, visit http://creativecommons.org/licenses/by-nc$\mathrm{nd} / 4.0 /$ 